\title{
On nowhere weakly symmetric functions and functions with two-element range
}

\author{
by
}

\author{
Krzysztof Ciesielski (Morgantown, WV), \\ Kandasamy Muthuvel (Oshkosh, WI) and \\ Andrzej Nowik (Sopot and Gdańsk)
}

\begin{abstract}
A function $f: \mathbb{R} \rightarrow\{0,1\}$ is weakly symmetric (resp. weakly symmetrically continuous) at $x \in \mathbb{R}$ provided there is a sequence $h_{n} \rightarrow 0$ such that $f\left(x+h_{n}\right)=$ $f\left(x-h_{n}\right)=f(x)$ (resp. $f\left(x+h_{n}\right)=f\left(x-h_{n}\right)$ ) for every $n$. We characterize the sets $S(f)$ of all points at which $f$ fails to be weakly symmetrically continuous and show that $f$ must be weakly symmetric at some $x \in \mathbb{R} \backslash S(f)$. In particular, there is no $f: \mathbb{R} \rightarrow\{0,1\}$ which is nowhere weakly symmetric.

It is also shown that if at each point $x$ we ignore some countable set from which we can choose the sequence $h_{n}$, then there exists a function $f: \mathbb{R} \rightarrow\{0,1\}$ which is nowhere weakly symmetric in this weaker sense if and only if the continuum hypothesis holds.
\end{abstract}

1. Introduction. The terminology of this note is standard and follows [5].

Recall that a function $f: \mathbb{R} \rightarrow \mathbb{R}$ is symmetrically continuous provided

$$
\lim _{h \rightarrow 0}[f(x+h)-f(x-h)]=0
$$

for every $x \in \mathbb{R}$. A "total negation" of the condition (1) leads to the notion of nowhere weakly symmetrically continuous function $\left({ }^{1}\right)$, that is,

$$
\lim _{n \rightarrow \infty}\left[f\left(x+h_{n}\right)-f\left(x-h_{n}\right)\right] \neq 0
$$

for every sequence $h_{n} \searrow 0$ and all $x \in \mathbb{R}$. It is easy to see that if the range of $f$ is discrete then (2) is equivalent to: " $f\left(x+h_{n}\right) \neq f\left(x-h_{n}\right)$ for all

2000 Mathematics Subject Classification: Primary 26A15; Secondary 03E50, 03E35.

Key words and phrases: continuum hypothesis, second category sets, two-element range functions, symmetric functions, symmetrically continuous functions, anti-Schwartz functions.

$\left(^{1}\right)$ This terminology comes from a monograph by Thomson [12]. Ciesielski and Larson [7] and most other papers on this subject call such a function uniformly antisymmetric. 
but finitely many $n . "$ In particular, if $f: \mathbb{R} \rightarrow \mathbb{N}$ then $f$ is not weakly symmetrically continuous at $x \in \mathbb{R}$ provided $f(x+h) \neq f(x-h)$ for all $h>0$ small enough, that is, when there exists an $\varepsilon_{x}>0$ such that the interval $\left(0, \varepsilon_{x}\right)$ is disjoint from the set

$$
S_{x}=\{h>0: f(x-h)=f(x+h)\} .
$$

In [7] Ciesielski and Larson constructed a nowhere weakly symmetrically continuous function $f: \mathbb{R} \rightarrow \mathbb{N}$ for which each set $S_{x}$ is finite. (See also [5, Cor. 7.4.2].) They also show there that there is no nowhere weakly symmetrically continuous function with two-element range. The latter fact was proved earlier by Kostyrko [10].

Nowhere weakly symmetrically continuous functions have been studied by many authors. Komjáth and Shelah [9] investigated under what set theoretical assumptions the sizes of all sets $S_{x}$ can be bounded by a fixed number. (See also [4].) In [2] Ciesielski proved that there is no nowhere weakly symmetrically continuous function with three-element range and in [3] he showed that the technique of [2] cannot be used to prove that there is no nowhere weakly symmetrically continuous function with four-element range. It is still an open question (see [7, Problem 1(a)], [6, Problem 2(a)], or [12]) whether there exists a nowhere weakly symmetrically continuous function with finite range, though Ciesielski and Shelah [8] constructed a nowhere weakly symmetrically continuous function with bounded countable range.

For $f: \mathbb{R} \rightarrow \mathbb{R}$ let $S(f)$ denote the set of all points at which $f$ is not weakly symmetrically continuous, that is,

$$
S(f)=\{x \in \mathbb{R}: f \text { is not weakly symmetrically continuous at } x\} .
$$

We say that a set $A \subset \mathbb{R}$ is locally symmetric provided for every $x \in A$ there exists an $\varepsilon_{x}>0$ such that

$$
x-h \in A \Leftrightarrow x+h \in A \quad \text { for every } h \in\left(0, \varepsilon_{x}\right) .
$$

Our first theorem characterizes the family of all sets $S(f)$ with $f$ : $\mathbb{R} \rightarrow\{0,1\}$.

Theorem 1. (a) If $f: \mathbb{R} \rightarrow\{0,1\}$ then the set $S(f)$ is countable and locally symmetric.

(b) For every countable and locally symmetric subset $A$ of $\mathbb{R}$ there exists an $f: \mathbb{R} \rightarrow\{0,1\}$ such that $S(f)=A$.

A notion dual to weakly symmetrically continuous function is that of a symmetric function: a function $f: \mathbb{R} \rightarrow \mathbb{R}$ is symmetric provided

$$
\lim _{h \rightarrow 0}[f(x+h)+f(x-h)-2 f(x)]=0
$$

for every $x \in \mathbb{R}$. The "total negation" of (3) parallel to that done for the case of symmetrically continuous function leads to the notion of nowhere weakly 
symmetric function and was introduced by Ciesielski in [3] $\left({ }^{2}\right)$. We will be interested in this notion only for functions $f: \mathbb{R} \rightarrow S$ when $S \subset \mathbb{N}$ contains no arithmetic progression of length three. In this particular case it is more convenient to adopt a simpler equivalent definition: a function $f: \mathbb{R} \rightarrow S$ is nowhere weakly symmetric provided for every $x \in \mathbb{R}$ there exists an $\varepsilon_{x}>0$ such that the interval $\left(0, \varepsilon_{x}\right)$ is disjoint from the set

$$
T_{x}=\{h>0: f(x-h)=f(x)=f(x+h)\} .
$$

The paper [3] contains also a construction of a nowhere weakly symmetric function $f: \mathbb{R} \rightarrow S \subset \mathbb{N}$ for which $T_{x}=\emptyset$ for every $x \in \mathbb{R}$. It also contains the question whether there exists a nowhere weakly symmetric function with two-element range. One of the goals of this paper is to answer this question in the negative:

THEOREM 2. There is no nowhere weakly symmetric function $f$ : $\mathbb{R} \rightarrow\{0,1\}$.

In the second part of the paper we will consider the variations of weak symmetric continuity and weak symmetry associated with any ideal $\mathcal{I}$ on $\mathbb{R}$ in the following way: for every $x \in \mathbb{R}$ we choose an exceptional set $E_{x} \in \mathcal{I}$, and for this $x$ we consider only the sequences $h_{n}$ disjoint from $E_{x}$. Investigation of these notions for the ideal of countable subsets of $\mathbb{R}$ leads to the following theorem.

Theorem 3. The following conditions are equivalent.

(i) The Continuum Hypothesis.

(ii) There exists a function $f: \mathbb{R} \rightarrow\{0,1\}$ such that $\left|S_{x}\right| \leq \omega$ for every $x \in \mathbb{R}$.

(iii) There exists a function $f: \mathbb{R} \rightarrow\{0,1\}$ such that for every $x \in \mathbb{R}$ there exists an $\varepsilon_{x}>0$ such that $\left|S_{x} \cap\left(0, \varepsilon_{x}\right)\right| \leq \omega$.

(iv) There exists a function $f: \mathbb{R} \rightarrow\{0,1\}$ such that $\left|T_{x}\right| \leq \omega$ for every $x \in \mathbb{R}$.

(v) There exists a function $f: \mathbb{R} \rightarrow\{0,1\}$ such that for every $x \in \mathbb{R}$ there exists an $\varepsilon_{x}>0$ such that $\left|T_{x} \cap\left(0, \varepsilon_{x}\right)\right| \leq \omega$.

It should be pointed out here that the equivalences (i) $\Leftrightarrow($ ii $) \Leftrightarrow($ iii $)$ are known and were proved in [7]. (See also [5, Thm. 7.4.4].)

We also show (Theorems 9 and 10) that for the ideal of meager subsets of $\mathbb{R}$ a similar theorem cannot be proved.

$\left({ }^{2}\right)$ Ciesielski [3] calls such functions anti-Schwartz, while current terminology follows Thomson [12]. 


\section{Every function with two-element range is somewhere weakly symmetric}

Proof of Theorem 1(a). We first show that $S(f)$ is countable. For $0<n<\omega$ let

$$
S_{n}(f)=\left\{x \in \mathbb{R}:(0,1 / n) \cap S_{x}=\emptyset\right\} .
$$

Then clearly

$$
S(f)=\bigcup_{0<n<\omega} S_{n}(f) .
$$

We will show that each $S_{n}(f)$ is discrete, so countable.

Fix an $n<\omega, n>0$. We will show that if $a, b \in S_{n}(f)$ are such that $0<b-a<1 / n$ then the points $a$ and $b$ are isolated in $S_{n}(f)$. This clearly implies that $S_{n}(f)$ is discrete.

So take $a, b \in S_{n}(f)$ such that $0<b-a<1 / n$ and consider the set

$$
Z=\left(b-\frac{1}{n}, a+\frac{1}{n}\right) \backslash\{a, b\} .
$$

We will show that $Z \cap S_{n}(f)=\emptyset$, so that $a$ and $b$ are isolated in $S_{n}(f)$.

Thus, take any $z \in Z$. Since the diameter of $\{a, b, z\}$ is less than $1 / n$ we have

$$
f(2 a-z)=1-f(z)=f(2 b-z) .
$$

But the mid point $(a+b)-z$ of $2 a-z$ and $2 b-z$ is at distance less than $1 / n$ from $\{2 a-z, 2 b-z\}$. So $(a+b)-z \notin S_{n}(f)$.

This implies that the set $Z=(a+b)-Z$ is disjoint from $S_{n}(f)$, as required.

It is also easy to see that if $x \in S(f)$ and $\varepsilon_{x}>0$ is such that $\left(0, \varepsilon_{x}\right) \cap S_{x}$ $=\emptyset$ then

$$
x-h \in S(f) \Leftrightarrow x+h \in S(f) \quad \text { for every } h \in\left(0, \varepsilon_{x}\right) .
$$

Thus, $S(f)$ is locally symmetric.

Proof of Theorem 1(b). The proof presented here is an elaboration of that for $[9$, Thm. 1], where it is proved that for any countable subset $A$ of $\mathbb{R}$ there exists an $f: \mathbb{R} \rightarrow\{0,1\}$ with $A \subset S(f)$.

Let $\left\{a_{n}: 0<n<\omega\right\}$ be an enumeration of $A$ and $\left\{d_{n}: 0<n<\omega\right\}$ be an enumeration of $G(A) \backslash A$, where $G(A)$ is the additive group generated by $A$. Following [9] for every $n<\omega$ we define inductively a finite collection $\mathcal{I}_{n}=\left\{I_{\gamma}: \gamma \in \Gamma_{n}\right\}$ of open intervals such that $\emptyset=\Gamma_{0} \subseteq \Gamma_{1} \subseteq \ldots$, each $I_{\gamma}$ is of the form $\left(b_{\gamma}-h_{\gamma}, b_{\gamma}+h_{\gamma}\right)$, and the following properties hold for any $\gamma, \gamma^{\prime} \in \Gamma_{n}$ :

(1) If $\gamma \neq \gamma^{\prime}$, then either $I_{\gamma} \cap I_{\gamma^{\prime}}=\emptyset$ or one of the intervals $I_{\gamma}$ and $I_{\gamma^{\prime}}$ is a subset of the other.

(2) If $I_{\gamma^{\prime}} \subseteq I_{\gamma}$, then either $I_{\gamma^{\prime}} \subseteq\left(b_{\gamma}-h_{\gamma}, b_{\gamma}\right)$ or $I_{\gamma^{\prime}} \subseteq\left(b_{\gamma}, b_{\gamma}+h_{\gamma}\right)$. 
(3) $\left\{a_{1}, \ldots, a_{n}\right\} \subseteq B_{n} \subseteq A$, where $B_{n}=\left\{b_{\gamma}: \gamma \in \Gamma_{n}\right\}$.

(4) $b_{\gamma} \pm h_{\gamma} \notin A$.

(5) If $I_{\gamma^{\prime}} \subseteq I_{\gamma}$, then $\varphi_{\gamma}\left[I_{\gamma^{\prime}}\right] \in \mathcal{I}_{n}$, where $\varphi_{\gamma}: I_{\gamma} \rightarrow I_{\gamma}$ is the reflection with respect to $b_{\gamma}$, that is, $\varphi_{\gamma}(x)=2 b_{\gamma}-x$.

(6) If $x \in I_{\gamma}$ then $x \in A$ if and only if $\varphi_{\gamma}(x) \in A$.

(7) If $I_{\gamma} \notin \mathcal{I}_{n-1}$ then $d_{n} \notin I_{\gamma}$.

Conditions (1)-(5) are present in the proof of [9, Thm. 1], while (6) and (7) are specific to our construction.

The construction is pretty much a repetition of that in [9]. We start with $\Gamma_{0}=\mathcal{I}_{0}=\emptyset$. If $\mathcal{I}_{n-1}$ is already constructed, we construct $\mathcal{I}_{n}$ as follows. If $a_{n} \in B_{n-1}$ we put $\mathcal{I}_{n}=\mathcal{I}_{n-1}$. So assume that $a_{n} \notin B_{n-1}$ and notice that the set $B$ of all (well defined) numbers $\varphi_{\gamma_{1}} \circ \ldots \circ \varphi_{\gamma_{r}}\left(a_{n}\right)$ has at most $2^{\left|\mathcal{I}_{n-1}\right|}$ elements and that $a_{n} \in B \subset A \backslash B_{n-1}$. Find an $h>0$ small enough that for every $b \in B$ the interval $(b-h, b+h)$ is disjoint from the set $\left\{d_{1}, \ldots, d_{n}\right\} \cup B_{n} \cup\left\{b_{\gamma} \pm h_{\gamma}: \gamma \in \Gamma_{n-1}\right\}$ and that $A \cap(b-h, b+h)$ is symmetric with respect to $b$. Moreover, decreasing $h$ if necessary, we also require that $\{b \pm h: b \in B\}$ is disjoint from $A$. We define $\mathcal{I}_{n}$ as $\mathcal{I}_{n-1} \cup\{(b-h, b+h)$ : $b \in B\}$. It is easy to see that $\mathcal{I}_{n}$ is as desired.

In what follows we will write $y=\varphi_{\gamma_{1}} \circ \ldots \circ \varphi_{\gamma_{r}}(x)$ only when all $\gamma_{i}$ 's are from $\Gamma=\bigcup_{n<\omega} \Gamma_{n}$ and the value of the composition is well defined. Note also that if $y=\varphi_{\gamma_{1}} \circ \varphi_{\gamma_{2}}(x)$ and $I_{\gamma_{1}} \subsetneq I_{\gamma_{2}}$ then we also have $y=\varphi_{\gamma_{2}} \circ \varphi_{\gamma_{1}^{\prime}}(x)$, where $b_{\gamma_{1}^{\prime}}=\varphi_{\gamma_{2}}\left(b_{\gamma_{1}}\right)$. From this and the fact that $\varphi_{\gamma} \circ \varphi_{\gamma}(z)=z$ for every $z$ we can conclude that

(*) If $y=\varphi_{\gamma_{1}^{\prime}} \circ \ldots \circ \varphi_{\gamma_{s}^{\prime}}(x)$ there exists an $r \leq s$ of the same parity as $s$ and $\gamma_{1}, \ldots, \gamma_{r} \in \Gamma$ such that $y=\varphi_{\gamma_{1}} \circ \ldots \circ \varphi_{\gamma_{r}}(x)$ and $I_{\gamma_{1}} \supsetneq \ldots \supsetneq I_{\gamma_{r}}$.

Notice also the following two facts:

(A) If $y=\varphi_{\gamma_{1}} \circ \ldots \circ \varphi_{\gamma_{r}}(x)$ and $r$ is odd then $y \neq x$.

Indeed, by $(*)$ we can assume that $I_{\gamma_{1}} \supsetneq \ldots \supsetneq I_{\gamma_{r}}$. But this means that $x$ and $y$ are on opposite sides of $b_{\gamma_{1}}$, so they are not equal.

(B) For every $x_{0} \in \mathbb{R} \backslash A$ there exists a $\delta=\delta_{x}>0$ such that for every $x \in\left(x_{0}-\delta, x_{0}+\delta\right)$ if $y=\varphi_{\gamma_{1}} \circ \ldots \circ \varphi_{\gamma_{r}}(x)$ and $r$ is odd then $y \neq 2 x_{0}-x$.

To see this, first notice that

$$
y=\varphi_{\gamma_{1}} \circ \ldots \circ \varphi_{\gamma_{r}}(x)=-x-2 \sum_{i=1}^{r}(-1)^{i} b_{q_{i}} .
$$

Now, if $2 x_{0}-x=y=\varphi_{\gamma_{1}} \circ \ldots \circ \varphi_{\gamma_{r}}(x)$ then $x_{0}=-\sum_{i=1}^{r}(-1)^{i} b_{q_{i}} \in G(A)$. In particular, if $x_{0} \notin G(A)$ then (B) holds for any $\delta>0$. Thus, we can assume that $x_{0} \in G(A) \backslash A$, that is, $x_{0}=d_{n}$ for some $n<\omega$. Note also that 
if $x_{0}$ does not belong to any $I_{\gamma}$ then $y$ stays on the same side of $x_{0}$ that $x$ does and, once again, any $\delta>0$ works. So we can assume that $x_{0}$ belongs to some $I_{\gamma}$ which, by (7), must belong to $\mathcal{I}_{n-1}$.

Let $I_{\gamma}$ be the unique shortest interval such that $x_{0} \in I_{\gamma}$ and let $\delta>0$ be such that $\left(x_{0}-\delta, x_{0}+\delta\right) \subset\left(b_{\gamma}-h_{\gamma}, b_{\gamma}+h_{\gamma}\right) \backslash\left\{b_{\gamma}\right\}$. We will show that this $\delta$ satisfies (B).

Assume, by way of contradiction, that there is an $x \in\left(x_{0}-\delta, x_{0}+\delta\right)$ such that $2 x_{0}-x=y=\varphi_{\gamma_{1}} \circ \ldots \circ \varphi_{\gamma_{r}}(x)$ and $r$ is odd. By $(*)$ we can also assume that $I_{\gamma_{1}} \supsetneq \ldots \supsetneq I_{\gamma_{r}}$.

Since $b_{q_{1}}$ stays on one side of each $I_{q_{i}}, 1<i \leq r$, the points $x$ and $\varphi_{\gamma_{2}} \circ$ $\ldots \circ \varphi_{\gamma_{r}}(x)$ are on the same side of $b_{q_{1}}$. So $x$ and $2 x_{0}-x=\varphi_{\gamma_{1}} \circ \ldots \circ \varphi_{\gamma_{r}}(x)$ are on opposite sides of $b_{q_{1}}$. But $x, 2 x_{0}-x \in\left(x_{0}-\delta, x_{0}+\delta\right) \subset I_{\gamma}$, implying that $b_{q_{1}} \in\left(x_{0}-\delta, x_{0}+\delta\right) \subset I_{\gamma}$. So, $I_{\gamma_{1}} \subsetneq I_{\gamma}$. However, $x$ and $2 x_{0}-x=y$ also belong to $I_{\gamma_{1}}$, and so does $x_{0}$, which is between $x$ and $2 x_{0}-x$. This gives $x_{0} \in I_{\gamma_{1}} \subsetneq I_{\gamma}$, contradicting the choice of $I_{\gamma}$. This completes the proof of (B).

With (A) and (B) in hand, we can construct the desired function $f$. For this, consider $\mathbb{R}$ as vertices of an infinite graph with numbers $x$ and $y$ connected by an edge provided $y=\varphi_{\gamma}(x)$ for some $\gamma \in \Gamma$. Also, for each $x \in \mathbb{R}$ let $C_{x}$ stand for all $y \in \mathbb{R}$ such that there exists a path from $x$ to $y$, that is, $y=\varphi_{\gamma_{1}} \circ \ldots \circ \varphi_{\gamma_{r}}(x)$ for some $\gamma_{1}, \ldots, \gamma_{r} \in \Gamma$.

Note that condition (A) says that our graph has no odd cycles, so we can color it by two colors, 0 and 1 , such that no connected vertices have the same color. In fact, if we color each component $C_{x}$ that way, then the resulting coloring, a function $f: \mathbb{R} \rightarrow\{0,1\}$, will have this property. It is easy to see that for any such $f$ we will have $A \subset S(f)$. To see that we can find such an $f$ with $A=S(f)$, first note that, by (4), if $y \in C_{x}$ then $y$ belongs to the group generated by $A \cup\{x\}$. Thus each component $C_{x}$ is at most countable. We color components $C_{x}$ by transfinite induction. We enumerate $(\mathbb{R} \backslash A) \times \omega$ as $\left\langle\left\langle x_{\xi}, n_{\xi}\right\rangle: \xi<\mathfrak{c}\right\rangle$. Next, at each stage $\xi<\mathfrak{c}$ we choose and color two new components $C_{\xi}$ and $C_{\xi}^{\prime}$ in the following way. First we choose $x \in\left(x_{\xi}, x_{\xi}+\delta_{x_{\xi}} / 2^{n_{\xi}}\right)$ such that neither $x$ nor $2 x_{\xi}-x$ is in the previously chosen components. Then we choose components $C_{\xi}$ and $C_{\xi}^{\prime}$ containing $x$ and $2 x_{\xi}-x$, respectively, and color each in such a way that both $x$ and $2 x_{\xi}-x$ get the same color. Such a coloring can obviously be chosen if the components $C_{\xi}$ and $C_{\xi}^{\prime}$ are not equal. However, if $C_{\xi}=C_{\xi}^{\prime}$ then, by (B), $x$ and $2 x_{\xi}-x$ must get the same color automatically.

It is easy to see that the coloring function $f$ defined that way is as desired.

The characterization given in Theorem 1 does not hold for functions with a 3-element range. This follows from the fact that, by [3, Thm. 2], for every 
Hamel basis $H \subset \mathbb{R}$ there exists an $f: \mathbb{R} \rightarrow\{0,1,2\}$ such that $H \subset S(f)$. Since a Hamel basis can be a Bernstein set (see e.g. [5, Cor. 7.3.7]) the set $S(f)$ for such an $f$ needs to be neither meager nor of measure zero.

With Theorem 1 in hand we are ready to prove our second theorem.

Proof of Theorem 2. By way of contradiction assume that $f$ is nowhere weakly symmetric.

From Theorem 1 we know that $|S(f)| \leq \omega$. Translating $f$ if necessary, we can assume that $0 \notin S(f)$. Let $V$ be an uncountable linear subspace of $\mathbb{R}$ over $\mathbb{Q}$ disjoint from $S(f)$. We will obtain a contradiction with [7, Thm. 2.1] by showing that the restriction function

$$
f \nmid V: V \rightarrow\{0,1\}
$$

is nowhere weakly symmetrically continuous.

So, fix an $x \in V$. Since $f$ is not weakly symmetric at $x$ there exists an $\varepsilon>0$ such that $(0, \varepsilon) \cap T_{x}=\emptyset$. We will show that this implies that we also have $(0, \varepsilon) \cap S_{x} \cap V=\emptyset$. We assume that

$$
f(x)=0,
$$

the argument for $f(x)=1$ being essentially the same.

By way of contradiction assume that there is an $h \in(0, \varepsilon) \cap S_{x} \cap V$. Then $f(x-h)=f(x+h)$ is not equal to $f(x)=0$ since $h \notin T_{x}$. In particular,

$$
f(x-h)=f(x+h)=1 .
$$

Since $f$ is not weakly symmetric at $x+h$ and at $x-h$ we can find a $\delta \in(0, \varepsilon-h)$ such that $\left(T_{x-h} \cup T_{x+h}\right) \cap(0, \delta)=\emptyset$.

Since $x-h \in V \subset \mathbb{R} \backslash S(f)$ there exists a $t \in(0, \delta) \cap(0, h) \cap S_{x-h}$. Then $f((x-h)-t)=f((x-h)+t) \neq f(x-h)=1$, since $t \in S_{x-h} \backslash T_{x-h}$. Thus,

$$
f((x-h)-t)=f((x-h)+t)=0 .
$$

Since $h+t \in(0, \varepsilon) \subset \mathbb{R} \backslash T_{x}$ and $f((x-h)-t)=f(x)=0$ we conclude that

$$
f((x+h)+t)=1 .
$$

Similarly $h-t \in(0, \varepsilon) \subset \mathbb{R} \backslash T_{x}$ and $f((x-h)+t)=f(x)=0$ imply that

$$
f((x+h)-t)=1 \text {. }
$$

Thus $f((x+h)+t)=f((x+h)-t)=f(x+h)=1$, contradicting the fact that $t \notin T_{x+h}$.

Now for $f: \mathbb{R} \rightarrow \mathbb{R}$ let $T(f)$ be the set of all $x \in \mathbb{R}$ such that $f$ is not weakly symmetric at $x$. In this notation Theorem 2 says that $T(f) \neq \mathbb{R}$ for every function $f: \mathbb{R} \rightarrow\{0,1\}$. One might suspect that a characterization similar to that from Theorem 1 may also be true for $T(f)$. This, however, is not true. 
Example 4. There exists an $f: \mathbb{R} \rightarrow\{0,1\}$ such that $T(f)$ is not meager.

Proof. Let $f_{0}: \mathbb{R} \rightarrow \mathbb{N}$ be the function from [7, Cor. 1.2]. For this function none of the level sets $f_{0}^{-1}(n)$ contains an arithmetic progression of length 3 . Let $n \in \mathbb{N}$ be such that $f_{0}^{-1}(n)$ is not meager and let $f$ be the characteristic function of the set $f_{0}^{-1}(n)$. It is easy to see that $f_{0}^{-1}(n) \subset T(f)$.

Clearly, the same argument also gives a function $f: \mathbb{R} \rightarrow\{0,1\}$ for which the set $T(f)$ is not of measure zero.

The following fact is also worth mentioning here.

Proposition 5 (Szyszkowski [11]). For every $B \subset \mathbb{R}$ there exists an $f: \mathbb{R} \rightarrow \mathbb{N}$ for which $S(f)=B$.

We do not know whether its weakly symmetric analog is also true. Therefore the following question remains open.

Problem 6. Is it true that for every $B \subset \mathbb{R}$ there exists an $f: \mathbb{R} \rightarrow \mathbb{N}$ for which $T(f)=B$ ?

3. Nowhere almost weakly symmetric function with two-element range. We start this section with the proof of Theorem 3. Since the equivalences (i) $\Leftrightarrow($ ii) $\Leftrightarrow($ iii) are known it remains to show (i) $\Leftrightarrow($ iv $) \Leftrightarrow(\mathrm{v})$. This follows immediately from the following theorem.

TheOREM 7. Let $\kappa$ be an infinite cardinal number and let $W$ be a linear subspace of $\mathbb{R}$ over $\mathbb{Q}$. The following conditions are equivalent.

(i) $|W| \leq \kappa^{+}$.

(ii) There exists a function $f: \mathbb{R} \rightarrow\{0,1\}$ such that $\left|T_{x} \cap W\right| \leq \kappa$ for every $x \in W$.

(iii) There exists a function $f: \mathbb{R} \rightarrow\{0,1\}$ such that for every $x \in W$ there exists an $\varepsilon_{x}>0$ such that $\left|T_{x} \cap W \cap\left(0, \varepsilon_{x}\right)\right| \leq \kappa$.

We will prove Theorem 7 only for $\kappa=\omega$ which is enough to get Theorem 3. The proof of the general case is essentially the same. (Though it also requires a simple generalization of the first part of Theorem 3.) So, in the rest of the argument we assume that $\kappa=\omega$.

Since $T_{x} \subset S_{x}$, the implication (i) $\Rightarrow$ (ii) follows from the first part of Theorem 3. Clearly (ii) implies (iii). Thus, it is enough to prove that (iii) implies (i).

In the proof we will need the following lemma.

Lemma 8. Let $f: \mathbb{R} \rightarrow\{0,1\}$ and let $V$ be a linear subspace of $\mathbb{R}$ over $\mathbb{Q}$. If $Z=\left\{x \in V: \exists \varepsilon>0\left(\left|S_{x} \cap V \cap(0, \varepsilon)\right| \leq \omega\right)\right\}$ then $|Z| \leq \omega_{1}$. 
Proof. The proof is essentially identical to that for (iii) $\Rightarrow$ (i) from Theorem 3 as presented in [5, Thm. 7.4.4]. Just assume, by way of contradiction, that $|Z|>\omega_{1}$ and choose $B \subset Z$ of cardinality $\omega_{2}$ which is linearly independent over $\mathbb{Q}$. For this choice of $B$ the intact proof presented in [5, Thm. 7.4.4] gives the desired contradiction.

Proof of Theorem 7. Let $f$ be as in (iii) and, by way of contradiction, assume that $|W| \geq \omega_{2}$. Let $Z_{0}$ be as in Lemma 8 with $V=W$. By shifting the domain of $f$ if necessary, we can assume that $0 \notin Z_{0}$. Let $V$ be a linear subspace of $W$ over $\mathbb{Q}$ such that $|V| \geq \omega_{2}$ and $V \cap Z_{0}=\emptyset$. Apply again Lemma 8 to get a set $Z$ working for $f$ and this $V$. Then $|V \backslash Z| \geq \omega_{2}$.

Fix an $x \in V \backslash Z$. By (iii) we can choose an $\varepsilon>0$ with the property that $\left|T_{x} \cap W \cap(0,2 \varepsilon)\right| \leq \omega$. Since $x \notin Z$ we can also find an $h \in(0, \varepsilon) \cap S_{x} \cap V \backslash T_{x}$. In particular $f(x-h)=f(x+h)$. Next, assume that

$$
f(x)=0,
$$

the argument for $f(x)=1$ being essentially the same. Then

$$
f(x-h)=f(x+h)=1,
$$

since $h \notin T_{x}$. Let $\delta \in(0, h)$ be such that the set $T=\left(T_{x-h} \cup T_{x+h}\right) \cap W \cap(0, \delta)$ is countable. Since $x-h \in V \subset W \backslash Z_{0}$, the set $S_{x-h} \cap W \cap(0, \delta)$ is uncountable. In particular, there exists a $t \in S_{x-h} \cap W \cap(0, \delta) \backslash T$ such that

$$
h+t, h-t \in(0,2 \varepsilon) \backslash T_{x} .
$$

Then $f((x-h)-t)=f((x-h)+t) \neq f(x-h)=1$, since $t \in S_{x-h} \backslash T_{x-h}$. Thus,

$$
f((x-h)-t)=f((x-h)+t)=0 .
$$

Since $h+t \notin T_{x}$ and $f((x-h)-t)=f(x)=0$ we conclude that

$$
f((x+h)+t)=1 \text {. }
$$

Similarly $h-t \notin T_{x}$ and $f((x-h)+t)=f(x)=0$ imply that

$$
f((x+h)-t)=1 .
$$

Thus $f((x+h)+t)=f((x+h)-t)=f(x+h)=1$, contradicting the fact that $t \notin T_{x+h}$.

Let us finish with a remark that Theorem 7 can be proved with $W$ being replaced by any additive subgroup of $\mathbb{R}$.

The above theorems show that, under $\mathrm{CH}$, for a function $f: \mathbb{R} \rightarrow\{0,1\}$ the sets $S_{x}$ and $T_{x}$ can be countable. Can we make them any smaller? Can they all be, for example, nowhere dense? The following two theorems show that this is not possible. In what follows $\mathcal{M}_{0}$ and $\mathcal{M}$ will stand for the ideals of nowhere dense and meager subsets of $\mathbb{R}$, respectively. 
Theorem 9. Let $f: \mathbb{R} \rightarrow\{0,1\}, M \in \mathcal{M}$, and $V$ be a linear subspace of $\mathbb{R}$ over $\mathbb{Q}$. If $S_{x}^{M}=\left\{h \in S_{x} \cap V: h \notin \pm(x-M)\right\}$ and

$$
Z=\left\{x \in V: \exists \varepsilon>0\left(S_{x}^{M} \cap(0, \varepsilon) \in \mathcal{M}_{0}\right)\right\}
$$

then $Z \in \mathcal{M}$.

Proof. By way of contradiction assume that $Z \notin \mathcal{M}$. So, we also have $V \notin \mathcal{M}$. For every $x \in Z$ let $n_{x} \in \mathbb{N}$ be such that the set

$$
C_{x}=S_{x}^{M} \cap\left(0,1 / n_{x}\right)
$$

is nowhere dense. The sets $Z_{n}=\left\{x \in Z \backslash M: n_{x}=n\right\}$ form a countable partition of $Z \backslash M \notin \mathcal{M}$, so there exists an $n \in \mathbb{N}$ such that $Z_{n} \notin \mathcal{M}$. Let $U$ be an open interval of length less than $1 / n$ such that the set $L=Z_{n} \cap U$ is nowhere meager in $U$. Note that $n_{x}=n$ for all $x \in L$. Moreover, if

$$
C_{x}^{*}=C_{x} \cup \pm(x-M) \in \mathcal{M}
$$

then $S_{x} \cap V \cap(0,1 / n) \subset C_{x}^{*}$ so that

$$
f(x-h) \neq f(x+h) \quad \text { for } x \in L \text { and } h \in(0,1 / n) \cap V \backslash C_{x}^{*},
$$

and

$$
|x-y|<1 / n \quad \text { for every } x, y \in L .
$$

Let $x=t_{0} \in L$ and let $\left\{t_{m}: 0<m<\omega\right\}$ be a dense subset of $L \backslash\left(x \pm C_{x}^{*}\right)$. Moreover, let $K$ be an additive subgroup generated by $\left\{t_{m}: m<\omega\right\}$ and choose a

$$
z \in L \backslash \bigcup_{i<\omega}\left(K \pm C_{t_{i}}^{*}\right)
$$

$z \neq x$. Moreover, choose $y=t_{m} \notin\{x, z\}$ such that

$$
y \notin x \pm C_{z} .
$$

Such a choice is possible since $C_{z}$ is nowhere dense and $t_{m}$ 's are dense in $U$. Next define

$$
a=x-y+z, \quad b=x+y-z, \quad c=-x+y+z .
$$

Then $(a+b) / 2=x,(b+c) / 2=y$, and $(c+a) / 2=z$. We show that

$$
f(a) \neq f(b), \quad f(b) \neq f(c), \quad f(c) \neq f(a),
$$

which gives us the desired contradiction, since the points $a, b$, and $c$ are distinct and $f$ admits only two values. But (8) follows immediately from (5) and

$$
|a-x| \notin C_{x}^{*}, \quad|b-y| \notin C_{y}^{*}, \quad|c-z| \notin C_{z}^{*},
$$

since we obviously have $|a-x|,|b-y|,|c-z| \in(0,1 / n) \cap V$. But (9) follows easily from (6) and (7): 
$|a-x| \notin C_{x}^{*}$, since otherwise we would have $z-y=a-x \in \pm C_{x}^{*}$ and $z \in y \pm C_{x}^{*}$, contradicting (6).

$|b-y| \notin C_{y}^{*}$, since otherwise we would have $z-x=y-b \in \pm C_{y}^{*}$ and $z \in x \pm C_{y}^{*}$, again contradicting (6).

$|c-z| \notin C_{z}^{*}$, since otherwise the point $y-x=c-z$ would belong to the set $\pm C_{z}^{*}= \pm\left[C_{z} \cup \pm(z-M)\right]$. However, $y-x \in \pm C_{z}$ implies that $y \in x \pm C_{z}$, contradicting (7). On the other hand if $y-x \in \pm(z-M)$, then $z \in K+M$, contradicting (6).

Theorem 10. Let $f: \mathbb{R} \rightarrow\{0,1\}$. If

$$
U=\left\{x \in \mathbb{R}: \exists \varepsilon>0\left(T_{x} \cap(0, \varepsilon) \in \mathcal{M}_{0}\right)\right\}
$$

then $\mathbb{R} \backslash U \notin \mathcal{M}$.

Proof. The proof is quite similar to that for Theorem 7. By way of contradiction assume that $U^{\mathrm{c}}=\mathbb{R} \backslash U \in \mathcal{M}$. Let $Z_{0} \in \mathcal{M}$ be from Theorem 9 used with $V=\mathbb{R}$ and $M=\emptyset$. Then apply again Theorem 9 with $V=\mathbb{R}$ and $M=Z_{0} \cup U^{\mathrm{c}}$ to get a set $Z \in \mathcal{M}$.

Next fix an $x \in \mathbb{R} \backslash\left(Z \cup U^{\mathrm{c}}\right)$. We assume that

$$
f(x)=0,
$$

the argument for $f(x)=1$ being essentially the same. Since $x \in U$ we can choose an $\varepsilon>0$ such that $T_{x} \cap(0, \varepsilon) \in \mathcal{M}_{0}$. Since $x \notin Z$ we have $S_{x}^{M} \cap(0, \varepsilon)$ $\notin \mathcal{M}_{0}$. Thus, there exists a non-empty open interval $J_{0} \subset(0, \varepsilon) \backslash T_{x}$ intersecting $S_{x}^{M}=S_{x} \backslash \pm(x-M)$. Let $h \in S_{x}^{M} \cap J_{0}$. Then

$$
f(x-h)=f(x+h)=1,
$$

since $h \in S_{x} \backslash T_{x}$. Since $h \notin \pm(x-M)$ we have $x \pm h \in \mathbb{R} \backslash M=U \backslash Z_{0}$. So there exists a $\delta>0$ such that the set $T=\left(T_{x-h} \cup T_{x+h}\right) \cap(0, \delta)$ is nowhere dense. Decreasing $\delta$ if necessary, we can also assume that $J=(h-\delta, h+\delta) \subset J_{0}$. Next, since $x-h \notin Z_{0}$, we can find a $t \in S_{x-h} \cap(0, \delta) \backslash T$.

Then $f((x-h)-t)=f((x-h)+t) \neq f(x-h)=1$, since $t \in S_{x-h} \backslash T_{x-h}$. Thus,

$$
f((x-h)-t)=f((x-h)+t)=0 .
$$

But $h \pm t \in J \subset J_{0} \subset(0, \varepsilon) \backslash T_{x}$ and $f(x-(h \pm t))=f(x)=0$ imply that

$$
f(x+(h \pm t))=1 \text {. }
$$

Thus $f((x+h) \pm t)=f(x+h)=1$, contradicting the fact that $t \notin T_{x+h}$.

\section{References}

[1] F. Bagemihl, Independent sets and second category, Order 7 (1990), 65-66. 
[2] K. Ciesielski, On range of uniformly antisymmetric functions, Real Anal. Exchange 19 (1993-94), 616-619.*

[3] -, Uniformly antisymmetric functions and $K_{5}$, ibid. 21 (1995-96), 147-153.*

[4] -, Sum and difference free partitions of vector spaces, Colloq. Math. 71 (1996), 263-271.*

[5] - Set Theory for the Working Mathematician, London Math. Soc. Stud. Texts 39, Cambridge Univ. Press, 1997.

[6] —, Set theoretic real analysis, J. Appl. Anal. 3 (1997), 143-190.*

[7] K. Ciesielski and L. Larson, Uniformly antisymmetric functions, Real Anal. Exchange 19 (1993-94), 226-235.*

[8] K. Ciesielski and S. Shelah, Uniformly antisymmetric function with bounded range, ibid. 24 (1998-99), 615-619.*

[9] P. Komjáth and S. Shelah, On uniformly antisymmetric functions, ibid. 19 (1993-94), 218-225.

[10] P. Kostyrko, There is no strongly locally antisymmetric set, ibid. 17 (1991-92), 423-425.

[11] M. Szyszkowski, Points of weak symmetric continuity, ibid. 24 (1998-99), 807-813.

[12] B. Thomson, Symmetric Properties of Real Functions, Marcel Dekker, 1994.

Department of Mathematics

West Virginia University

Morgantown, WV 26506-6310, U.S.A.

E-mail: K_Cies@math.wvu.edu

Web: http://www.math.wvu.edu/ ${ }^{\sim}$ kcies

Department of Mathematics

University of Wisconsin-Oshkosh

Oshkosh, WI 54901-8601, U.S.A.

E-mail: muthuvel@vaxa.cis.uwosh.edu
Current address: Institute of Mathematics Polish Academy of Sciences Abrahama 18 Sopot, Poland

Permanent address: Department of Mathematics Gdańsk University Wita Stwosza 57 80-952 Gdańsk, Poland E-mail: matan@paula.univ.gda.pl Web: http://math.univ.gda.pl/ ${ }^{\sim}$ tm/a.nowik

Received 14 May 2000; in revised form 4 August 2000

*A preprint is available in electronic form from Set Theoretic Analysis Web Page: http://www.math.wvu.edu/ ${ }^{\sim}$ kcies/STA/STA.html 